\title{
D Department of
}

Journal of Rehabilitation Research and Development Vol. 27 No. 2, 1990

Pages 107-114

\section{Metabolic responses to arm ergometry and functional neuromuscular stimulation}

\author{
Bennett G. Edwards, PhD, RKT, and E.B. Marsolais, MD, PhD \\ VA/Medical Center, Cleveland, $\mathrm{OH} 44106$
}

\begin{abstract}
The hips and lower extremities of four complete paraplegic male subjects (T-6, T-7, T-8, and T-11) were stimulated with functional neuromuscular stimulation (FNS) via transcutaneous intramuscular electrodes $(20 \mathrm{~mA}, 0-150$ pulse width, and $20 \mathrm{~Hz}$ ). Cardiopulmonary (CP) and/or cardiovascular (CV) responses were measured during maximal (seated) arm ergometry (AE), FNS, and FNS + AE. Subjects' lower extremities were stimulated with a $2 \mathrm{~s}$ walking cycle via a microprocessor computer. Data were collected with a SensorMedic MMC Horizon ( $\dot{\mathrm{V}} \mathrm{O}_{2}$ and $\dot{\mathrm{V}} \mathrm{CO}_{2}$ at STPD and $\dot{\mathrm{VE}}$ at BPTS). The mean MET level $(1 \mathrm{MET}=3.5 \mathrm{ml} \mathrm{O} 2 / \mathrm{kg} / \mathrm{min}$ ) during FNS was 4.8. Mean METS during FNS + AE was 10.3 and mean METS for AE was 7.2. Mean lactic acid (LA) after FNS, AE, and FNS + AE was $73 \mathrm{mg}$ percent, $77 \mathrm{mg}$ percent and $115 \mathrm{mg}$ percent respectively. Respiratory exchange ratio (RER) $\left(\dot{\mathrm{V} C O} \mathrm{C}_{2} / \dot{\mathrm{VO}}_{2}\right)$ was $>1.2$ during the first 2 to 5 min of FNS but decreased to $<1.0$ during the second 5 min of FNS. Steady state $\dot{\mathrm{V}} \mathrm{O}_{2}$ and RER $<1.0$ indicated a FNS transition from anaerobic to aerobic metabolism. Subject $\mathrm{T}-11$ had $\mathrm{CV}$ limitations during FNS and FNS + AE due to excessive LA from FNS (115 mg percent). Ventilatory ( $\dot{V} E$ ) responses during AE, FNS, and FNS $+\mathrm{AE}$ were consistent with $\mathrm{VO}_{2}$; and mean maximal $\dot{\mathrm{VE}}$ and $\dot{\mathrm{V}} \mathrm{O}_{2}$ for subjects $\mathrm{T}-6, \mathrm{~T}-7$, and $\mathrm{T}-8$ during FNS + AE was greater than 90 percent of that observed in sedentary normals. The aerobic and anaerobic capacities of paraplegic subjects is primarily limited by available muscle mass rather than impaired $\mathrm{CV}$ or CP function.
\end{abstract}

Key words: aerobic and anaerobic metabolism, cardiopulmonary and cardiovascular responses, functional neuromuscular

Address all correspondence and reprint requests to: Bennett G. Edwards, PhD, Department of Community Health and Rehabilitation, Norfolk State University, 2401 Corprew Avenue, Norfolk, VA 23504. stimulation, microprocessor computer, paraplegia, respiratory exchange ratio.

\section{INTRODUCTION}

It has been demonstrated that paraplegic subjects who walk with FNS via percutaneous intramuscular electrodes (17) are unable to sustain their walking activity for more than 30 to 300 meters distance due to cardiovascular fatigue and/or perceived exertion $(11,17)$. Since FNS walking involves both arm and leg work, it is necessary to determine maximal aerobic power (MAP and/or maximal oxygen consumption) for FNS (hips and lower extremities), arm ergometry (AE), and AE + FNS in order to isolate the various components that make up the total and relative energy costs of FNS walking. Analysis of three paraplegic subjects during FNS walking showed that their aerobic energy costs were approximately six times greater than their basal metabolism (17). Stimulation of these subjects with FNS walking parameters (supine), while emulating their average speeds of FNS walking, resulted in aerobic energy costs that were three times their basal metabolism (17).

Normal sedentary individuals have been able to sustain muscular activity at or below 50 percent of their MAP (2). However, FNS may have a high anaerobic component -at or below 50 percent MAP-due to synchronized stimulation of motor neurons and muscle fibers. In addition, surface functional electrical stimulation (FES) tends to recruit large superficial motor neurons (fast twitch) more efficiently than deeper and smaller (slow twitch) motor neurons (3). This type of stimulation promotes the 
accumulation of metabolites and fatigue at the neuromuscular junction despite stimulation frequencies that usually recruit slow twitch fibers in normal muscle (3). When paralyzed and normal muscle were stimulated with surface FES both accumulated lactic acid (LA), but the accumulation was much greater in paralyzed muscle (upper motor neuron lesion), possibly due to its deconditioned state (5).

The purpose of this study was to determine whether open-loop FNS (preset parameters with no feedback) could substantially increase the MAP and LA accumulation in paraplegic subjects when combined with maximal $\mathrm{AE}$.

\section{METHODS}

After reading and signing informed consent papers, four complete paraplegic subjects (T-6 to T-11) stimulated their lower extremities with percutaneous intramuscular electrodes (20 mA, 0-150 microsecond pulse width and 20 pps) over a period of 2 to 4 years; an average of 1 hour per day 4 times a week. All subjects were males, ages 23, 26, 28, and 40. A portable microprocessor-controlled stimulator (19) delivered FNS during actual FNS walking, with a rolling walker, and seated FNS walking to the following muscles: vastus lateralis, vastus intermedius, vastus medialis, tensor fasciae latae, sartorius, gracilis, semimembranosus, posterior portion of adductor magnus, gluteus maximus and medius, soleus and tibialis anterior. Electrodes were implanted with needles next to the motor points of the involved muscles (18). Subject data are presented in Table 1.

Seated FNS exercise consisted of 10 min of a $2 \mathrm{~s}$ walking cycle ( $0.6 \mathrm{~s}$ stance and $0.4 \mathrm{~s}$ swing) with a $20 \mathrm{lb}$ weight around each ankle to limit leg movement for safety purposes. Subjects were stabilized in their wheelchairs by straps around their pelvis and lower abdominals. The AE protocol consisted of a 2 min exercise, $15 \mathrm{~s}$ rest, and weight adjusted test (21). RPM was maintained at 50 and rhythm was maintained with a metronome. Subjects stopped

Table 1.

Subject data.

\begin{tabular}{cccccc}
\hline Subject \# & Injury level & Age & Weight & HR-rest & BP-rest \\
1 & T-7 & 40 & $63 \mathrm{~kg}$ & 64 & $104 / 82$ \\
2 & T-8 & 26 & $75 \mathrm{~kg}$ & 80 & $122 / 84$ \\
3 & T-6 & 24 & $61 \mathrm{~kg}$ & 76 & $112 / 82$ \\
4 & T-11 & 28 & $77 \mathrm{~kg}$ & 68 & $110 / 82$ \\
\hline
\end{tabular}

exercise when they were no longer able to maintain AE rhythm.

Pulmonary data were collected with a SensorMedic Mobile Metabolic Cart (MMC). A standard room temperature was maintained and data were collected in the afternoon at least 2 hours after eating lunch. The MMC was calibrated by entering barometric pressure, gas checks, temperature checks, volume checks, and subject height and weight. A two-way face mask was placed over each subject's mouth and nose prior to testing. A Monark Rehabilitation Trainer was calibrated with a known weight prior to $\mathrm{AE}$ and combined $\mathrm{AE}+\mathrm{FNS}$ testing. Heart rate (HR) was monitored by a Life-Pak-5 and interfaced with the MMC to give $15 \mathrm{~s}$ average printouts and to calculate $\mathrm{O}_{2}$ pulse. Pulmonary data, $\dot{\mathrm{VO}} \mathrm{O}_{2}, \dot{\mathrm{V}} \mathrm{CO}_{2}, \mathrm{RER}\left(\mathrm{VCO}_{2} / \dot{\mathrm{VO}}_{2}\right.$, $) \mathrm{VE}$, METS (basal metabolic rate $=1 \mathrm{MET}=3.5 \mathrm{ml}$ $\mathrm{O}_{2} / \mathrm{kg} / \mathrm{min}$ ), and breath frequency were monitored until they became stable. Blood pressures (BP) were obtained at the beginning and end of the exercise protocols and during the $15 \mathrm{~s}$ breaks with $\mathrm{AE}$ whenever possible. Lactic acid (LA) was determined immediately before and 5 min after each protocol from readings obtained from a YSI-23 Lactate Analyzer. The net difference between initial and end LA was recorded. Blood for LA was obtained from a finger prick and placed in a tube containing centrimonium bromide to lyse the cells to obtain total blood lactate. The lactate analyzer was calibrated with standard solutions and tested for linearity prior to assays. Subject warm-up was avoided to observe all transitional responses and to keep LA within the standard range of the analyzer $(0-15 \mathrm{mM})$. All data were collected on separate days from one of the three protocols: FNS, AE, FNS + AE (Table 2).

Four repeated measures of AE + FNS by subject 2 showed no more than \pm 1 percent difference between trials for the data obtained (MAP and LA). Maximal results were obtained from AE only when the subject sat erect with his arms able to fully extend the ergometer pedals. His shoulders had to be the same height as the pedals. This procedure was adopted and used for all of the subjects. Care was taken not to strap the subjects around their upper abdominals because this caused labored breathing and lowered AE results.

\section{RESULTS}

The mean MET level obtained during FNS was 47 percent (FNS/FNS + AE) of that obtained from FNS + $\mathrm{AE}$ and the range was between 26 and 72 percent. Mean net LA accumulation 5 min post-FNS exercise was 63 
percent of that obtained 5 min post-FNS + AE (range 27 to 89 percent), All subjects had higher $\mathrm{O}_{2}$ pulse for FNS + AE compared to that obtained from FNS or AE alone, as shown in Table 2 . Three of the four subjects obtained their highest rate pressure product (RPP) during AE. The mean maximal RER was 1.3 during AE prior to exhaustion (see AE power outputs in Table 3). Mean maximal RER during FNS was 1.4 (range 1.2 to 2.0 ) during the first $5 \mathrm{~min}$ of FNS but it dropped to 0.9 during the second 5 min of FNS (range 0.8 to 1.0 ). Mean maximal RER during the first 2 to 5 min of FNS + AE was 1.4 (range 1.2 to 1.7 ), but had a mean of 1.3 prior to exhaustion (range 1.2 to 1.4). It should be noted that RER was recorded during exercise activity and not during the $15 \mathrm{~s}$ AE breaks. Mean systolic blood pressure was $+6 \mathrm{~mm} \mathrm{Hg}$ higher during FNS compared to AE + FNS.

\section{DISCUSSION}

\section{Muscle strength and endurance}

Preliminary data indicated that muscular strength and endurance were directly related to the frequency, duration, and magnitude of muscle spasms rather than the number of years of stimulation. Muscle spasms were evaluated clinically (standard muscle testing) and by patient interview. Isokinetic data (Cybex II) indicated that maximal strength and endurance were attained by individually implanted muscles within 90 days (10). Relatively weaker muscles (quadriceps) maintained 50 percent of their maximal force and range of motion much longer than stronger muscles (10). Strength and endurance variations in stronger muscles made it necessary to use weaker muscles whenever possible. It should be noted that most stronger muscles were actually combinations of various quadriceps muscles rather than individual muscles. Isokinetic Cybex data (one leg) at 60 degrees per $\mathrm{s}$ showed that all four subjects reached 50 percent peak torque and 50 percent total work after 2 to $5 \mathrm{~min}$ of FNS quadriceps exercise (10). The fatigue time course closely followed the transition to steady state $\dot{\mathrm{VO}}_{2}$ during FNS walking (seated) exercise.

\section{Hemodynamics}

Collins and Glaser (8) used closed-loop (feedback) surface FES to exercise the quadriceps muscles (20 lb maximal resistance on each leg) of two quadriplegic and two paraplegic subjects and compared their metabolic responses to four normal subjects. They noted higher HR, VE, and $\mathrm{VO}_{2}$ for the disabled subjects compared to the normal subjects when exercising at the same workloads. They suggested that the average FNS HR responses (11 bpm below resting levels) were inadequate and that FES had a high anaerobic component due to impaired sympathetic nervous system responses, which ultimately resulted in reduced muscle blood flow. However, blood $\mathrm{pH}$ or blood lactate were not reported in this study. Each exercise period consisted of $7 \mathrm{~s}$ alternated contractions ( 2 per min for each leg) over a 4 min period.

The FNS walking program in this study allowed rhythmic contractions of all the muscles involved in FNS walking. There were approximately 60 contractions per min (30 per leg). Since blood tends to pool in the lower extremities of some paraplegics due to the lack of muscle tone and vasomotor tone, elevated $\mathrm{HR}$ at rest may be the result of decreased venous return (4). FNS + AE utilized a large muscle mass whose pumping action, from repeated contractions, increased venous return. This affected the preload pressure receptors, resulting in increased HR and cardiac output (Brainbridge reflex). This was substantiated by the fact that $\mathrm{O}_{2}$ pulse, an indicator of stroke volume, was much larger during FNS + AE than during AE or FNS alone. In addition, there were linear increases in HR, BP, and $\mathrm{O}_{2}$ pulse during FNS. Heart rate and BP responses during FNS and FNS + AE were consistent with the aerobic workload encountered (7). RPP indicated that three of the four subjects had greater myocardio-consumption during AE compared to FNS or FNS + AE. In general, this was expected since $\mathrm{AE}$ in normal subjects elicits higher HR and BP than leg work or arm and leg work requiring the same mechanical energy output $(2,12)$. Mean systolic BP was highest during FNS (+6 mmHg), possibly reflecting increased peripheral resistance due to near maximal individual muscle contractions (ischemic) initially incurred. Lower RER during the first 2 to $5 \mathrm{~min}$ of FNS + AE compared to the first 2 to $5 \mathrm{~min}$ of FNS only indicated that cardiopulmonary adjustments occurred more efficiently when a larger muscle mass was used.

\section{Lactic acid and RER}

Moderate intensity dynamic work in normal subjects elicits lactic acid accumulation for the initial 2 to $3 \mathrm{~min}$ before cardiovascular adjustments are sufficient to meet the workload aerobically. Since open-loop FNS elicits maximal synchronized contractions (normal muscles fire asynchronously), lactic acid may have accumulated for a longer period. This was substantiated by RERs $>1.2$ in all subjects observed during the first 5 min of FNS (subject 4 had a RER of 2.0). However, mean RER dropped to 0.9 during the second 5 min of FNS. This, in conjunction with steady state oxygen consumption, indicated a transition 
Journal of Rehabilitation Research and Development Vol. 27 No. 2 Spring 1990

Table 2.

Metabolic data.

\begin{tabular}{|c|c|c|c|}
\hline Subject \# & FNS & $\mathbf{F N S}+\mathbf{A E}$ & $\mathbf{A E}$ \\
\hline \multicolumn{4}{|l|}{ HR } \\
\hline 1 & 144 & 174 & 186 \\
\hline 2 & 118 & 186 & 182 \\
\hline 3 & 120 & 174 & 204 \\
\hline 4 & 140 & 150 & 198 \\
\hline \multicolumn{4}{|c|}{ METS $\left(3.5 \mathrm{ml} \mathrm{O} \mathrm{O}_{2} / \mathrm{kg} / \mathrm{min}\right)$} \\
\hline 1 & 5.9 & 10.4 & 5.3 \\
\hline 2 & 3.2 & 12.2 & 8.5 \\
\hline 3 & 5.5 & 11.9 & 8.9 \\
\hline 4 & 4.7 & 6.5 & 8.2 \\
\hline \multicolumn{4}{|c|}{ VE $1 /$ min BTPS } \\
\hline 1 & 51 & 95 & 70 \\
\hline 2 & 32 & 111 & 85 \\
\hline 3 & 42 & 84 & 73 \\
\hline 4 & 48 & 66 & 81 \\
\hline \multicolumn{4}{|c|}{$\dot{\mathrm{V} E} / \dot{\mathrm{V}} \mathrm{O}_{2}\left(\dot{\mathrm{V} E}\right.$ per 1 liter of $\left.\dot{\mathrm{V}} \mathrm{O}_{2}\right)$} \\
\hline 1 & 40 & 41 & 60 \\
\hline 2 & 39 & 35 & 38 \\
\hline 3 & 47 & 33 & 41 \\
\hline 4 & 59 & 47 & 38 \\
\hline \multicolumn{4}{|c|}{ LA mg/100ml } \\
\hline 1 & 77 & 95 & 50 \\
\hline 2 & 32 & 117 & 120 \\
\hline 3 & 67 & 120 & 61 \\
\hline 4 & 115 & 129 & 78 \\
\hline \multicolumn{4}{|l|}{ RPP } \\
\hline 1 & 219 & 216 & 260 \\
\hline 2 & 170 & 275 & 236 \\
\hline 3 & 156 & 226 & 265 \\
\hline 4 & 188 & 195 & 238 \\
\hline \multicolumn{4}{|l|}{$\mathrm{O}_{2}$ Pulse } \\
\hline 1 & 9.0 & 13.2 & 6.2 \\
\hline 2 & 7.0 & 16.5 & 12.2 \\
\hline 3 & 9.8 & 14.1 & 9.2 \\
\hline 4 & 9.0 & 11.0 & 10.8 \\
\hline
\end{tabular}

from anaerobic to aerobic metabolism. Respiratory exchange ratios in normal subjects rarely exceed 1.2 during maximal stress tests of any type, but RERs during supramaximal stress may be as high as 1.5 (13). Normal subjects adjust to progressively resistive workloads during stress tests and stop when they are no longer able to maintain that work rate (1). Workloads are reduced during activity with FNS, which can be demonstrated by the reduction of isokinetic quadriceps readings to 50 percent peak torque and 50 percent total work during the first 2 to 5 min of isokinetic exercise. Davies et al. (9) found the halftime (t-1/2) of $\mathrm{O}_{2}$ and $\mathrm{CO}_{2}$ from heavier to light work to be $45 \mathrm{~s}$ and $126 \mathrm{~s}$, respectively. Thus, a RER of 2.0 occurring in conjunction with a decreasing highly anaerobic FNS workload was not particularly unusual. The subject with the highest RERs had the highest lactic acid reading (subject 4) and vice-versa (subject 2).

Subject 4 had total blood LA accumulation of 115 $\mathrm{mg} / 100 \mathrm{ml}$ during FNS, which was consistent with maximal LA in sedentary normal subjects who performed 
Table 3.

Maximal arm power output.*

\begin{tabular}{cccrr}
\hline Subject \# & AE + FNS & AE & AE + FNS & AE \\
1 & 6 & 8 & 57 & 76 \\
2 & 10 & 12 & 113 & 135 \\
3 & 10 & 10 & 92 & 92 \\
4 & 6 & 10 & 69 & 116 \\
\hline
\end{tabular}

*Watts.

maximal stress tests using a large muscle mass (20). Mean MAP and maximal LA tolerance is usually much higher (2) when normal subjects use a large muscle mass (legs or arms and legs) compared to a small muscle mass (arms alone). When subject 4 attempted FNS + AE, his MET level was the lowest observed for all subjects during this activity. Since LA from muscles engaged in heavy anaerobic exercise affects the performance of nonexercising muscles, it appears that lactate uptake from the blood stream functions identically to endogenously formed LA (15). This was further evidenced by subject 4 achieving his highest $\dot{\mathrm{V}} \mathrm{O}_{2}$ during $\mathrm{AE}$ (higher than FNS + AE). The lowest LA observed post-FNS exercise was $32 \mathrm{mg} / 100$ $\mathrm{ml}$ (subject 2) and this was consistent with optimalstrenuous work done by normal sedentary subjects performing stress tests using a large muscle mass (20). Only subject 3 was able to match his maximal AE power output during FNS + AE (Table 3). The relatively high MAP of the arms of subjects 2 and 3 and the relatively low MAP and LA from FNS enabled these subjects to obtain MET levels during FNS + AE that were at or above 75 percent of normal subjects during standardized fitness tests (1).

\section{FNS and fatigue}

Clinical observations of FNS walking showed that subject 1 stopped because of arm fatigue, which was usually preceded by fatigue of the trunk and hip musculature. This was evidenced by complaints of arm pain and an excessive forward lean. Subject 1 had the lowest MAP and the lowest anaerobic power (expressed by $\dot{\mathrm{VO}}_{2}$ and LA, respectively) observed during $\mathrm{AE}$ in all subjects. Subject 4 stopped FNS walking because of frequent knee buckling. Maximal LA accumulation during FNS undoubtedly caused muscle fatigue and a subsequent reduction in quadriceps torque. Although subject 2 had the strongest quadriceps muscle torques observed, he fatigued (t-50 percent maximal peak torque) faster than the other subjects during isokinetic testing. During surface FES and internal FNS, stronger muscles appear to fatigue faster than weaker ones $(10,16)$.

FNS as a percentage of total MAP (less than 50 percent) does not seem to pose any limitations to FNS walking (with the highly probable exception of subject 4). Subject 4 was taking a calcium channel blocker to relax his bladder and this may have adversely affected the contractile mechanism of his muscles. In addition, his muscles were very strong, which resulted in increased LA and possible ischemia.

In normal subjects, maximal isometric contractions fall 50 percent in about $1 \mathrm{~min}$ and EMG indicates that high frequency motor neurons cease firing and low frequency motor neurons are recruited at this time (2). This suggests that neuromuscular junction (acetylcholine) fatigue occurs in normal subjects. During surface FNS, however, Benton et al. (3) reported neuromuscular junction fatigue at frequencies below $30 \mathrm{pps}$ (frequencies in this current study were 20 pps or below). Since FNS did not require prolonged work in the present study, energy substrates, muscle glycogen, or electrolyte imbalances were not the cause of local muscle fatigue. The most likely cause for local muscle fatigue in this study was ischemia. Cybex data indicated that FNS subjects could sustain isokinetic quadriceps contractions at 60 degrees per $\mathrm{s}$ for $10 \mathrm{~min}$ or more (more than 300 repetitions). Steady state $\dot{\mathrm{VO}}_{2}$ physiological responses to FNS closely resemble those of normal muscle performing 30 repetitions per min at 60 percent of maximal peak torques (2).

\section{Isolated energy cost during FNS}

Marsolais and Edwards (17) determined the mean MET level of three complete paraplegic subjects (T-4 to T-11), walking with FNS at speeds between 0.1 and $0.6 \mathrm{~m} / \mathrm{s}$ for 0.75 to $1.25 \mathrm{~min}$, to be between 5 and 6 METS. Subjects 2 and 4 in the present study participated in that study as well. By subtracting the energy of FNS from the energy costs of FNS walking, one can grossly estimate the energy consumed by the arms and upper torso during FNS walking. Subject 4 walked for $58 \mathrm{~min}(0.8 \mathrm{~s}$ per step, versus 
Journal of Rehabilitation Research and Development Vol. 27 No. 2 Spring 1990

$1.0 \mathrm{~s}$ per step during FNS exercise) with FNS and a rolling walker, and obtained a mean MET level of 5.5. By subtracting 4.7 METS (FNS) from 5.5 (FNS walking) the net aerobic work of the arms and torso during FNS walking was estimated to be 0.8 METS. The gross aerobic work rate can be estimated by adding 1.0 MET (basal metabolic rate) to the net to obtain 1.8 METS.

In the future, FNS exercise must correspond with the cadence and time of FNS walking. One can then subtract $\mathrm{O}_{2}$ consumption and LA during FNS exercise from that obtained during FNS walking to estimate the aerobic and anaerobic work done by the upper extremities and torso. This is possible because the magnitude, duration, and frequency of open-loop FNS remains constant. The percentage of MAP (AE) used by the upper body, percentage of MAP (FNS) used by the lower body, and percentage of total MAP (AE + FNS) can also be estimated. When METS for FNS and AE were added for subjects 1, 2, and 3 , they were 93 percent of that obtained during AE + FNS. High LA during FNS obviously limited subject 4 during $\mathrm{AE}+\mathrm{FNS}$. However, the popular belief that people with paraplegia have reduced total MAP due to impaired respiratory and abdominal muscles or compromised autonomic nervous systems has been refuted. With FNS training and AE many paraplegic persons can obtain MAP that is as high or higher than normal individuals.

Burdett et al. (6) compared several biomechanical methods for determining the energy costs of normal walking (center of mass, joint angles, ground reaction forces, body segments) and compared them to metabolic energy expenditure. They found correlations between 0.79 and 0.89 . Although the mechanical work during swing-through crutch walking was very similar to normal walking, the former had 30 percent greater metabolic energy costs (3). This was probably due to the fact that swing-through crutch ambulation requires one to perform isometric contractions, making it difficult to measure the mechanical work of cocontractors, fixators and stabilizers. Ground reaction forces (subject 2) obtained by strain gauges implanted in loftstrand crutches revealed that peak vertical forces during FNS walking (2-point gait) was 30 percent of body weight during stance and dropped to zero during swing.* This percentage of body weight can be tolerated indefinitely by most individuals (14).

\section{CONCLUSION}

Three of four subjects had MET levels during $10 \mathrm{~min}$ of FNS ( 2 s walking cycle) which were 26,46 , and 56 percent of that obtained during AE + FNS. An additional paraplegic subject (T-4), had METS during FNS that were 30 percent of that obtained during AE + FNS. The relatively low percentage of total MAP (AE + FNS), and LA (32 to $77 \mathrm{mg} / 100 \mathrm{ml}$ ) indicated that the metabolic costs of FNS does not limit FNS walking. Subject 4 had very high LA during FNS which resulted in general fatigue and local muscle fatigue (as exhibited by large reductions in torque and range of motion during isokinetic FNS quadriceps exercise). In this instance, FNS was limiting to FNS walking. If open-loop FNS is matched with the cadence and time of open-loop FNS walking, one may be able to isolate the metabolic costs of the upper and lower body during FNS walking. Mean METS for all subjects during FNS was less than 50 percent of that obtained during FNS + AE. This ergometry will help to screen and set up training regimes for candidates of FNS walking programs.

\section{ACKNOWLEDGMENT}

This work has been supported by the Department of Veterans Affairs, Rehabilitation Research and Development Service.

\section{REFERENCES}

1. American College of Sports Medicine: Guidelines for Exercise Testing and Prescription (3rd Edition). Philadelphia: Lea \& Febiger, 1986.

2. Astrand P, Rodahl K: Textbook of Work Physiology. New York, NY: McGraw-Hill Book Co., 1977.

3. Benton LA, Baker LL, Bowman BR, Waters RL: Functional Electrical Stimulation-A Practical Guide. Downey, CA: Rancho Los Amigos Hospital, 1981.

4. Berne RM, Levy MN: Cardiovascular Physiology. St. Louis, MO: C.V. Mosby Co., 1981.

5. Brice RM, Forster HV, Pan L, Funahashi A, Hoffaman M, Lowry T, Murphy C: Arterial $\mathrm{pH}$ and lactate during electrically induced leg exercise in paraplegic and normal subjects. Med Sci Sports Exerc 18(2):Supplement, 1986.

6. Burdett RG, Skirinar SG, Simon SR: Comparison of mechanical and metabolic energy consumption during normal gait. J Orthot Res 1:63-72, 1983.

7. Clausen JP: Circulatory adjustments to dynamic exercise and effect of training in normal subjects and in patients with coronary artery disease. Prog Cardiovasc Dis 18:459, 1976. 
8. Collins SR, Glaser RM: Comparisons of aerobic and cardiopulmonary responses for electrically induced and voluntary exercise. In Proceedings of the 8th Annual RESNA Conference, 391-393. Washington, DC: Association for the Advancement of Rehabilitation Technology, 1985.

9. Davies CTM, di Prampero PE, Cerretelli PE: Kinetics of cardiac output and respiratory gas exchange during exercise and recovery. $J$ Appl Physiol 32(5):618-625, 1972.

10. Edwards BG: Quadriceps muscle response to functional neuromuscular stimulation during isokinetic exercise and walking. In Proceedings of the 10th Annual RESNA Conference, 605-607. Washington, DC: Association for the Advancement of Rehabilitation Technology, 1987.

11. Edwards BG, Lew RD, Marsolais EB: Relative energy costs of long leg brace and FNS ambulation. In Proceedings of the 9th Annual RESNA Conference, 322-324. Washington, DC: Association for the Advancement of Rehabilitation Technology, 1986.

12. Figoni SF: Spinal cord injury and maximal aerobic power. Am Corr Ther J 38:44-50, 1984.

13. Issekutz B Jr, Birkhead NC, Rodahl K: Use of respiratory quotients in assessment of aerobic work capacity. $J$ Appl Physiol 17(1):47-50, 1962.

14. Jebsen RH: Use and abuse of ambulation aids. JAMA 199:63-68, 1967.
15. Karlson J, Peterson FB, Henrickson HG: Effects of previous exercise with arms and legs on metabolism and performance in exhaustive exercise. $J$ Appl Physiol 38(5):763-767, 1975.

16. Kralj A, Grabelnik S: Functional neuromuscular stimulation: A new hope for paraplegic patients? Bull Prosthet Res 51:182-201, 1973.

17. Marsolais EB, Edwards BG: Energy costs of walking and standing with functional neuromuscular stimulation and long leg braces. Arch Phys Med Rehabil 69:243-249, 1988.

18. Marsolais EB, Kobetic R: Implantation techniques and experiences with percutaneous intramuscular electrodes in the lower extremities. J Rehabil Res Dev 23(3):1-8, 1986.

19. Vance FR, Kobetic R, Chizeck H: Portable microprocessor controlled stimulator for the activation of paralyzed muscles. In Proceedings of the $23 \mathrm{rd}$ International Symposium for Mini and Micro Computers and Their Applications. San Antonio, TX, 1983.

20. Wells JG, Balke B, Van Fossan DD: Lactic acid accumulation during work: A suggested standardization of work classification. J Appl Physiol 10(1):51-55, 1957.

21. William J, Cottrell E, Powers SK, McKnight T: Arm ergometry. A review of published protocols and the introduction of a new weight adjusted protocol. $J$ Sports Med 23:107-112, 1983.

Authors' Notes: Discussion and Research Issues by Bennett G. Edwards, PhD, RKT, and E.B. Marsolais, MD, PhD

Blocker and Associates (1983) confirmed and documented the fact that spinal cord injury $(\mathrm{SCI})$ patients have increased coronary risk factors and/or abnormal electrocardiogram findings. The present study has demonstrated that functional neuromuscular stimulation (FNS), when combined with arm ergometry (AE), substantially increases the aerobic and anaerobic capacity of paraplegic subjects.

Can FNS + AE exercise reverse coronary risk factors in SCI patients? Longitudinal studies need to be conducted to determine the long-term health benefits of FNS + AE exercise. Can FNS + AE exercise prevent decubitus ulcers, reduce bone demineralization, increase longevity and quality of life? What are the potential risks? Do the risks outweigh the benefits? Would the use of AE with sur- face FNS electrodes, using similar muscles and stimulation patterns used during FNS in the present study, be more efficient and cost-effective?

Present data suggest that paraplegic subjects have sufficient aerobic and anaerobic capacity to walk several hundred meters with FNS and a rolling walker while maintaining a cardiopulmonary reserve. The problem seems to be local muscle fatigue due to FNS (resulting in torque reduction and/or loss of range of motion) and the amount of weight on the arms during ambulation. The latter problem has been partially alleviated by stimulating the erector spinae muscles and/or the use of trunk bracing. Since muscle training with FNS seems to have physiological limitations, the fatigue problem has been addressed by reducing the duty cycle (on/off ratio) and stimulation 
Journal of Rehabilitation Research and Development Vol. 27 No. 2 Spring 1990

parameters (frequency, pulse width, and amplitude) while increasing ambulation speed. Although open-loop specific task programming is a problem, the major problem is to develop a closed-loop control system that will respond quickly and safely to physiological and biomechanical changes during FNS ambulation.

These are just a few of the concerns and issues that need to be addressed in the field of AE + FNS exercise and FNS ambulation.

\section{REFERENCES}

Blocker WP, Merrill JM, Krebs MA, Cardus DP, Ostermann HJ: Electrocardiograph survey of patients with chronic spinal cord injury. Am Corr Ther J (July-August):101-104, 1983.

Marsolais EB, Edwards BG: Energy costs of walking and standing with functional neuromuscular stimulation and long leg braces. Arch Phys Med Rehabil 69:243-249, 1988. 